\title{
Tracking with Heavily Irradiated Silicon Detectors Operated at Cryogenic Temperatures
}

L. Casagrande ${ }^{1}$, B.M. Barnett ${ }^{2}$, P. Bartalini ${ }^{3}$, W.H. Bell ${ }^{4}$, K. Borer ${ }^{5}$, T. Bowcock ${ }^{6}$, J. Buytaert ${ }^{8}$, P. Chochula ${ }^{7}$, P. Collins ${ }^{8}$, C. Da Viá ${ }^{9}$, H. Dijkstra ${ }^{8}$, O. Dormond ${ }^{3}$, A. Esposito ${ }^{10}$, R. Frei $^{3}$, V. Granata ${ }^{11}$, S. Janos ${ }^{5}$, I. Konorov ${ }^{9}$, C. Lourenço ${ }^{8}$, T.O. Niinikoski ${ }^{8}$, S. Pagano ${ }^{11,12}$, V.G. Palmieri ${ }^{5}$, C. Parkes ${ }^{8}$, S. Paul ${ }^{9}$, K. Pretzl ${ }^{5}$, T. Ruf ${ }^{8}$, G. Ruggiero ${ }^{12}$, S. Saladino ${ }^{8}$, L. Schmitt ${ }^{9}$, K. Smith ${ }^{4}$, P. Sonderegger ${ }^{8}$, I. Stavitski ${ }^{13}$, D. Steele ${ }^{3}$, F. Vitobello ${ }^{11}$

${ }^{1}$ LIP, Lisbon, Portugal; ${ }^{2}$ Johannes-Gutenberg University, Mainz, Germany; ${ }^{3}$ University of Lausanne, Switzerland; ${ }^{4}$ Glasgow University, U.K.; ${ }^{5}$ LHEP, University of Bern, Switzerland; ${ }^{6}$ University of Liverpool, U.K.; ${ }^{7}$ HEPHY, Wien, Austria; ${ }^{8}$ CERN, Geneva, Switzerland; ${ }^{9}$ Technical University, Munich, Germany; ${ }^{10}$ ETL, Tsukuba, Japan; ${ }^{11}$ CNR, Arco Felice, Italy; ${ }^{12}$ INFN and University of Naples, Italy; ${ }^{13}$ INFN and University of Padova, Italy

\section{Abstract}

In this work we show that a heavily irradiated double-sided silicon microstrip detector recovers its performance when operated at cryogenic temperatures. A DELPHI microstrip detector, irradiated to a fluence of $\sim 4 \times 10^{14} \mathrm{p} / \mathrm{cm}^{2}$, no longer operational at room temperature, cannot be distinguished from a non-irradiated one when operated at $T<120 \mathrm{~K}$. Besides confirming the previously observed 'Lazarus effect' in single diodes, these results establish, for the first time, the possibility of using standard silicon detectors for tracking applications in extremely demanding radiation environments.

\section{INTRODUCTION}

The problem of radiation damage in silicon detectors is a key issue in view of their use in the high radiation environment of future high energy physics experiments. Moderate cooling is normally applied to irradiated silicon detectors, to lower the leakage current and to inhibit reverse annealing [1]. However, this kind of operation only delays bulk type inversion and does not prevent dramatic depletion voltage changes. Moreover, if the radiation fluence approaches $10^{15} \mathrm{n} / \mathrm{cm}^{2}$ (the expected fluence of the innermost trackers after 10 years of CERN-LHC operation [2]) the detectors become unusable [3].

Recent results have shown [4] that radiation induced defects in silicon can be electrically passivated if the device is operated at cryogenic temperatures (this is usually referred to as the 'Lazarus effect'). As a result, the Charge Collection Efficiency (CCE) is recovered to values similar to those achieved before irradiation, even for very high fluences. Furthermore, at cryogenic temperatures the signal becomes considerably faster due to the increased mobility of the carriers, while the leakage current becomes completely negligible [5]. These two features facilitate the readout front-end electronics design in terms of speed and noise requirements.

The measurements described in this paper were done to verify that the CCE recovery observed in heavily irradiated silicon detectors when operated at cryogenic temperatures also applies to segmented devices. Indeed, the recovered charge must remain focused, a mandatory requirement for tracking purposes.

\section{The LAZARUS EFFECT}

The charge collection inefficiency in an irradiated silicon detector is due both to incomplete depletion and to recombination or trapping of drifting charges in radiation-induced defects. While the first effect corresponds to a simple reduction in sensitive volume, the latter is more complicated. Empty traps in the space charge region can, in fact, capture electrons or holes, which are then released at a later time. Clearly, if the average de-trapping time is longer than the readout time, the charge carrier is lost. In irradiated detectors operated at room temperature it is possible to increase the CCE by using a very high bias voltage. However, this further increases the leakage current created by the damage, significantly degrading the signal to noise ratio.

The characterisation of radiation induced defects in silicon has shown [6] that at cryogenic temperatures it is still possible to effectively deplete an irradiated detector. Furthermore, most of the radiation induced traps are deep in the silicon forbidden band gap. Since at low temperatures the de-trapping rate of electrons and holes from deep levels is strongly affected by the reduced thermal energy, once a carrier is trapped, it cannot be emitted. Trapping of drifting charges becomes the predominant effect, leading to the condition that an important fraction of traps are filled, hence inactive. This prevents further trapping of carriers generated by particles traversing the detector and, therefore, no signal is lost. Similar properties have been observed in the case of diamond detectors, in which the as-grown material already contains very deep traps. In this case, even at room temperature, their neutralization is normally achieved by optical or electrical trap-filling [7]. It is important to stress that in the case of cryogenic operation, since the reverse annealing does not play a significant role, the devices only need to be cooled during operation and can otherwise be kept at room temperature.

From this picture, no conclusion can be derived for tracking devices. The condition that the CCE is recovered is not sufficient since the position resolution requires the additional feature that the particle signal is well focused. Therefore, it was important to verify the relevance of the Lazarus effect for tracking applications, by testing the performance of a heavily irradiated microstrip detector at cryogenic temperatures. 


\section{IRRADIATED MICROSTRIP TRACKER}

We tested a silicon tracker consisting of two planes of microstrip detectors operated at cryogenic temperatures. One plane was heavily irradiated, as explained below. We used DELPHI AC-coupled double-sided microstrips, with strips orthogonal to each other on opposite sides of the detector. The strip pitch on the p-side is $25 \mu \mathrm{m}$ ( $50 \mu \mathrm{m}$ readout pitch), while on the $\mathrm{n}$-side it is $42 \mu \mathrm{m}$. The detectors are glued to a $\mathrm{BeO}$ hybrid circuit where the readout chips are placed. The microstrips are wire-bonded to the input channels of the readout chips.

The readout chip (MX6), produced in $3 \mu \mathrm{m}$ CMOS technology, is a 128 input channels amplifier, with $1.5 \mu \mathrm{s}$ peaking time. A more detailed description of the DELPHI microstrip detectors can be found in Ref. [8]. It is worth mentioning that we had to remove the $\mathrm{BeO}$ rails normally glued to the edges of the detectors, in order to allow the silicon to accommodate the thermal stresses during the cooling step.

A complete characterisation of the tracker is beyond the purpose of this work. Nevertheless, it should be noticed that the results discussed in the following apply to a full module, consisting of a fairly large number of channels (1280).

\section{EXPERIMENTAL SET-UP}

The measurements were done in the X5 secondary muon beam of the CERN-SPS. The muon momentum was $100 \mathrm{GeV} / \mathrm{c}$. The experimental set-up is illustrated in Fig. 1 .

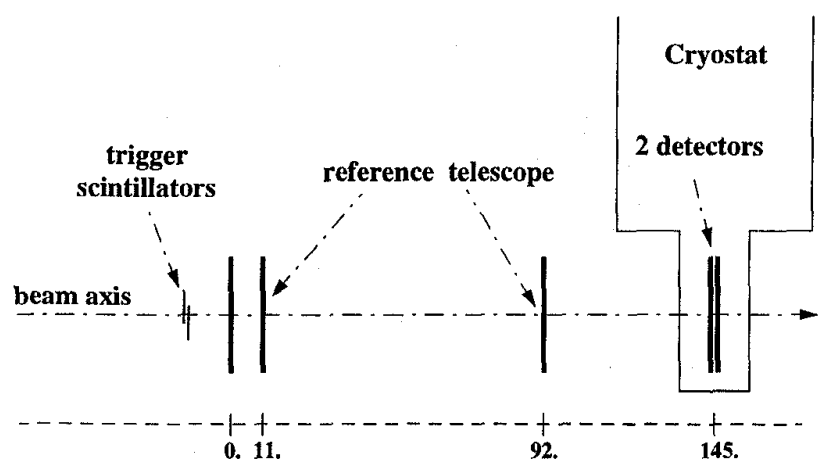

Figure 1: Schematic illustration of the experimental setup.

Two scintillators select muon tracks in the acceptance of the reference tracking telescope, which consists of 3 stations of silicon microstrip detectors. Each station measures four coordinates $\left(x, y, 45^{\circ}\right.$ and $\left.-45^{\circ}\right)$. The stations are built using single sided $25 \mu \mathrm{m}$ pitch microstrip detectors. A more detailed description of this telescope can be found in Ref. [9]. Downstream from the reference telescope sit the two DELPHI modules, placed inside a cryostat.

The irradiated plane was placed between the reference telescope and the non-irradiated one. The two DELPHI modules were mounted on a top loading cryogenic insert, sealed in a Oxford Variox BL beam cryostat that has a liquid $\mathrm{He}$ reservoir shielded by liquid $\mathrm{N}_{2}$. The integrated variable temperature volume extends into a tail below the main body of the cryostat, equipped with thin aluminized mylar windows. Liquid $\mathrm{He}$ from the main reservoir passes through a needle valve and via a capillary tube to a heat exchanger surrounding the central sample tube. The temperature is controlled by the dual action of regulating both the flow of liquid $\mathrm{He}$ and the electrical power in a resistor in the heat exchanger. The central sample tube contains low pressure $\mathrm{He}$ gas to cool the insert. The use of this particular cooling system was motived by practical constraints. Clearly, for operation at $77 \mathrm{~K}$ a much simpler approach based on liquid $\mathrm{N}_{2}$ can be used and no liquid $\mathrm{He}$ is required.

The DELPHI readout chips, despite having been designed for room temperature operation, were fully functional at cryogenic temperatures. This was expected from the well-known behaviour of CMOS circuits operated at cryogenic temperatures [10].

The second (non-irradiated) DELPHI plane is used as a reference to monitor the behaviour of the irradiated one. During the measurements, the temperatures of the detector chamber (thermal bath) and of the module hybrids were continuously monitored by means of platinum resistors. Due to the power dissipation of the readout electronics, the real temperature of the detector is somewhat lower than the hybrid temperature.

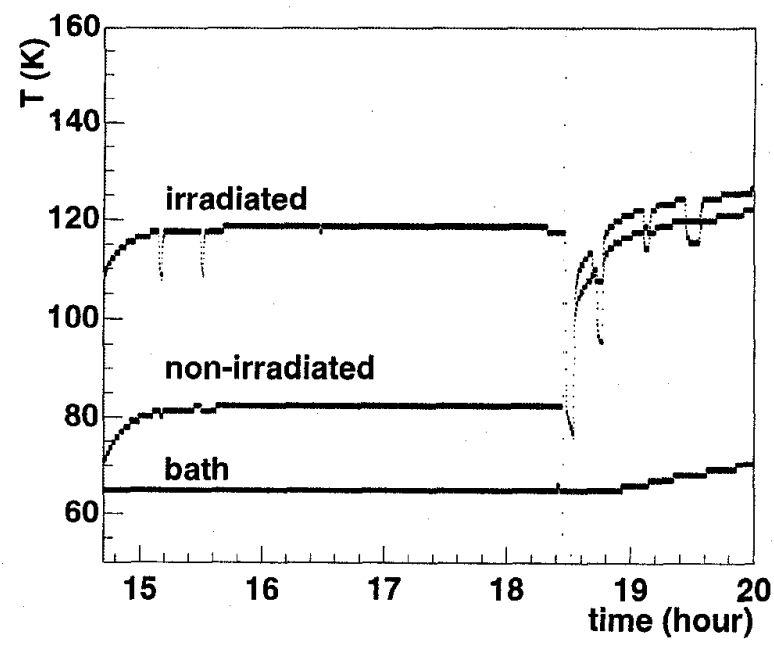

Figure 2: Bath and hybrids temperature evolution. At around $18 \mathrm{~h} 30$ the non-irradiated detector was switched on, doubling the power dissipated in the system.

It is worth mentioning that the 20 MX6 chips present inside the cryostat were dissipating over $10 \mathrm{~W}$. This resulted in a drastic temperature increase of the hybrids. A typical temperature profile evolution is shown in Fig. 2. During the test we had two running modes: the first with only the irradiated module powered and the second with both modules powered. With both modules switched-on it was not possible to keep their temperature constant. This was simply due to the small cooling power of our Oxford BL and does not reflect any significant constraint. 


\section{RESULTS}

The results presented in this paper correspond to a first study of the collected data. Further analysis is in progress and more detailed results will be available in the near future.

One of the two DELPHI detectors was irradiated with $24 \mathrm{GeV}$ protons at the CERN-PS. The area covered by the proton beam was around $5 \times 2 \mathrm{~cm}^{2}$. The maximum fluence was $\sim 4 \times 10^{14}$ protons $/ \mathrm{cm}^{2}$, reached in a geometrical region close to the edge of the detector ( $y \sim-2$ in Fig. 3). Since these detectors are built using a standard technology, the irradiated part showed a drastic leakage current increase after irradiation. The length of the module $(12 \mathrm{~cm})$ prevented radiation damage to occur at the location of the readout chips. Therefore, the radiation-soft electronics remained functional.

Despite the large noise due to the high leakage current, we could still perform an efficiency scan of the irradiated region, at room temperature, using a laser beam. A map of the measured laser signal is shown in Fig. 3. In this case the laser was shone on the p-side while measuring on the n-side. The inefficiency is probably due to the incomplete depletion at the maximum allowed voltage $(90 \mathrm{~V})$. This result clearly shows that most of the detector is no longer functional at room temperature.

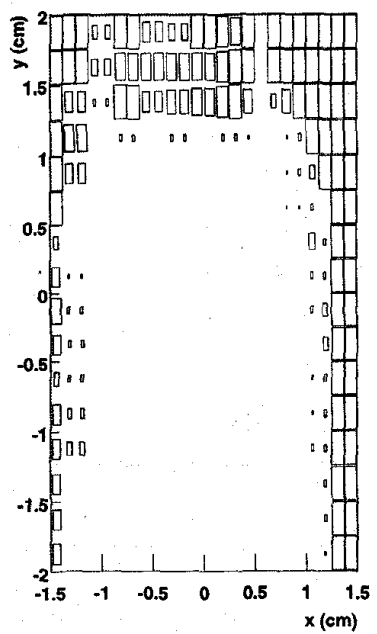

Figure 3: Laser scan of the irradiated detector, at room temperature.

The efficiency of the irradiated detector was measured by extrapolating the muon tracks reconstructed in the reference telescope. Figure 4 shows the profile of the extrapolated tracks, as selected by the trigger scintillators, in the irradiated detector plane. For the data analysis we selected the fiducial region where the trigger efficiency is uniform. This region matches that of interest on the irradiated detector. We computed the efficiency of the irradiated detector using the number of hits found in correspondence with extrapolated tracks.

The efficiency of the p-side of the irradiated detector for two different operating temperatures is shown in Fig. 5. In this case the bias voltage was set to $50 \mathrm{~V}$. These plots show that when the temperature of the hybrid is $\sim 120 \mathrm{~K}$ the detector is fully efficient, while at higher temperature an inefficient region

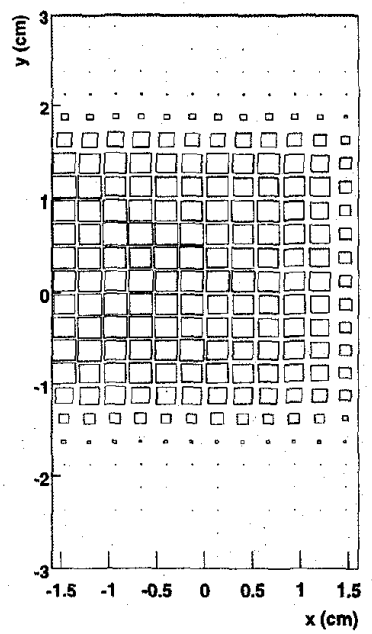

Figure 4: Map of the extrapolated tracks, at the $z$ position of the irradiated detector. The trigger efficiency is clearly visible.

appears in correlation with the irradiation spot.

The correct operation of the irradiated detector is confirmed by the cluster size analysis shown in Fig. 6. No significant difference is found between the irradiated and non-irradiated detectors.
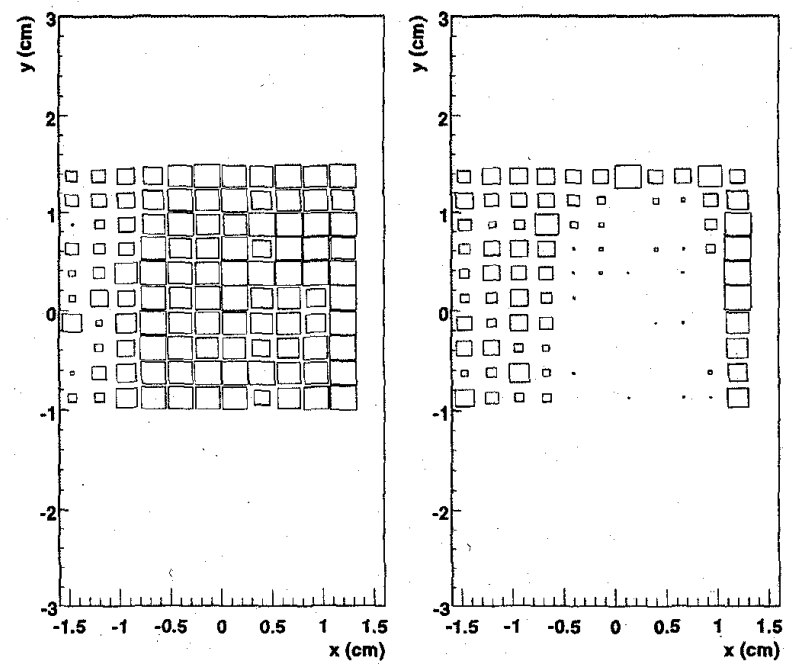

Figure 5: Detection efficiency of the irradiated plane, in the trigger fiducial region, for $V_{\mathrm{bias}}=50 \mathrm{~V}$, at $T=115 \mathrm{~K}$ (left) and at $T=130 \mathrm{~K}$ (right). The lower efficiency for $x<-1.2$ is due to malfunctioning of one readout chip.

From the pulse height distributions of the irradiated and non-irradiated detectors we conclude that the observed recovery in efficiency is actually due to a recovery in CCE, as previously observed in single diodes. Indeed, fitting the two Landau distributions we see that the most probable values differ by less than $10 \%$. These results clearly demonstrate that the irradiated detector, with a bias voltage of only $50 \mathrm{~V}$, cannot be distinguished from the non-irradiated one when operated at 


\section{ACKNOWLEDGMENTS}
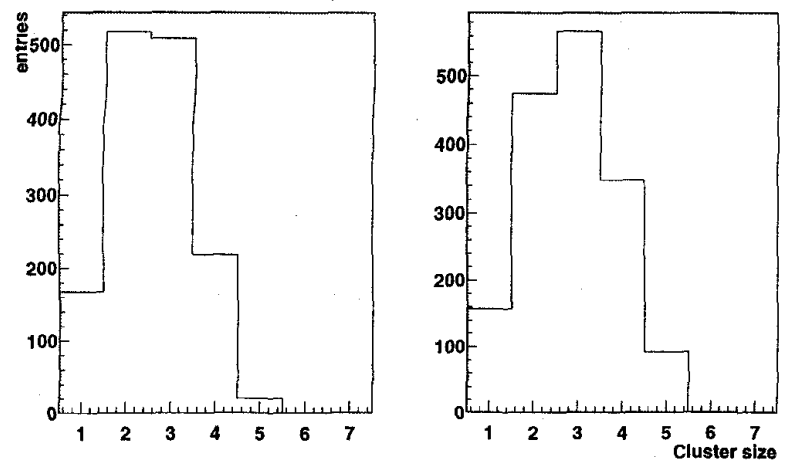

Figure 6: Cluster size distribution for the non-irradiated (left) and irradiated (right) detectors.

$T<120 \mathrm{~K}$. Since we know (see Ref. [4]) that the signal is fast $(<5 \mathrm{~ns}$ ), the results are not affected by the long shaping time used in these measurements.

\section{CONCLUSIONS}

We have shown that, when operated at cryogenic temperatures, a heavily irradiated double-sided silicon microstrip detector recovers its performance. This result is in agreement with what has been previously measured in single diodes. Moreover the fact that the detector is fully efficient at relatively low bias voltage shows that operation at cryogenic temperatures is a valid alternative to sophisticated technologies like special detector materials, or multi-guardring design for high voltage operation. No special care in the front-end part of the readout chip design is required due to the absence of leakage current. However, an optimization of CMOS readout circuits for operation at liquid nitrogen temperature could result in further improvements in terms of signal to noise ratio. It is worth stressing that the results presented here apply to a full module used in a real experiment and not just to a test device. This suggests that cryogenic operation of silicon detectors is a valid and reliable alternative for large tracking systems.

Despite the complexity of the set-up used in the experiment, a much simpler cooling approach can be used for operation of a silicon tracker at cryogenic temperatures. Work in the direction of a low mass system based on continuous two-phase flow of nitrogen in a capillary pipe is currently in progress in the RD39 collaboration. So far, all the factors suggest that silicon detectors operated at cryogenic temperatures could be the detectors of choice for the next generation of high luminosity particle physics experiments.
The work described in this paper resulted from a joint effort among people from the RD39 collaboration and the COMPASS, DELPHI and LHC-B experiments. We thank the IPHE-Lausanne, the LHEP-Bern and the CERN-EP Microelectronics workshops for technical support. We thank C. Buttar for helping with the irradiation in the CERN-PS, A. Hauska for participating in the setup preparation and S. Gerassimiov for helpful discussions concerning the reference telescope. One of the authors (L.C.) acknowledges financial support from the EC. (TMR programme) under contract number ERBFMBICT961204. This research was supported by the Schweizer Nationalfonds zur Foerderung der wissenschaftlichen Forschung (FORCE) and by the Stiftung zur Foerderung der wissenschaftlichen Forschung an der Universitaet Bern.

\section{REFERENCES}

[1] ATLAS Inner Detector TDR, CERN/LHCC/97-16/17, 1997; CMS Tracker Project TDR, CERN/LHCC/98-6, 1998.

[2] ATLAS Int. Note, INDET-NO-203, 1998.

[3] RD48 Collaboration Status Report, CERN/LHCC 98-39, 1998.

[4] V.G. Palmieri et al., Nucl. Instr. and Meth. in Phys. Res. A 413 (1998) 475.

[5] G. Ottaviani et al., Phys. Rev. B 12 (1975) 3318;

C. Canali et al., Phys. Rev. B 12 (1975) 2265.

[6] V. Eremin et al., Nucl. Instr. and Meth. in Phys. Res. A 372 (1996) 388;

Z. Li et al., Nucl. Instr. and Meth. in Phys. Res. A 388 (1997) 297.

[7] RD42 Collaboration Status Report, CERN/LHCC 98-39, 1998.

[8] V. Chabaud et al., Nucl. Instr. and Meth. in Phys. Res. A 368 (1996) 314.

[9] Nucl. Instr. and Meth. in Phys. Res. A 348 (1994) 444.

[10] Proceedings of the WOLTE Conference, Journ. de Phys. IV, Vol.8, 1998. 